Revista de Matemática: Teoría y Aplicaciones 1999 6(1) : 35-50

CIMPA - UCR - CCSS ISSN: 1409-2433

\title{
ANÁLISIS DISCRIMINANTE DESCRIPTIVO: TEORÍA, ALGORITMO Y SOFTWARE
}

\author{
William Castillo Elizondo*- Oldemar Rodríguez Rojas ${ }^{\dagger}$
}

Recibido: 23 Octubre 1998

\begin{abstract}
Resumen
El propósito de este artículo es presentar una implementación computacional del Análisis Discriminante Descriptivo. Para ello hemos desarrollado los aspectos teóricos que nos permiten formular el algoritmo que ha sido programado en $\mathrm{C}++$. Finalmente se ilustra el funcionamiento del software y el método, mediante un ejemplo.
\end{abstract}

Palabras clave: Análisis discriminante, algoritmo, variable discriminante, grupo a priori, baricentro.

\begin{abstract}
The objective of this article is to present a computational implementation of the Descriptive Discriminant Analysis. We have developed some theoretical aspects in order to formulate an algorithm that was implemented using $\mathrm{C}++$. Finally, the method and the software are illustrated by an example.
\end{abstract}

Keywords: Discrimination, algorithm, discriminant variable, a priori group, baricenter.

AMS Subject Classification: 62-07,62H30,68N99.

\section{Introdución}

Un problema de Análisis Discriminante (también decimos: un problema de discriminación) se presenta cuando es necesario 'explicar' una variable cualitativa con base en un cierto número de variables cuantitativas llamadas variables explicativas o predictores. Pero es

\footnotetext{
* CIMPA, Escuela de Matemática, Universidad de Costa Rica, 2060 San José, Costa Rica; Tel.: +(506) 2075574, Fax: +(506) 207 4397; E-Mail: wcastill@cariari.ucr.ac.cr

† CEREMADE, Université de Paris IX-Dauphine, Place du Maréchal de Lattre de Tassigny, 75775 París Cedex 16, Francia. E-Mail: orodrigu@ceremade.dauphine.fr
} 
bien conocido que existe una amplia gama de métodos para hacer discriminación en presencia de predictores cualitativos [1]. Uno de estos métodos, importante por sus aplicaciones, es el método 'Disqual' de G. Saporta usado para el cálculo de puntajes (credit scoring) [6]. El Análisis Discriminante se puede entender como un conjunto de métodos y procedimientos estadístico-matemáticos orientados a la búsqueda de dos objetivos, que pueden ser complementarios:

1. Determinar si las variables observadas permiten distinguir (discriminar) los $r$ grupos a priori. Este objetivo es de carácter descriptivo y se relaciona con el Análisis en Componentes Principales. Es natural entonces que se dé la mayor importancia a la construcción de representaciones bidimensionales de los individuos, de las variables y de los grupos a priori.

2. Construir reglas de clasificación -reglas decisionales- para asignar cada nuevo objeto a uno de los grupos a priori. Este objetivo es de carácter decisional y su nexo es con los métodos probabilísticos. Esencial a este énfasis es la construcción de reglas de decisión y los procedimientos para su evaluación

Este artículo trata el Análisis Discriminante solo en relación con el primer objetivo. Es decir, desde un punto de vista descriptivo, por eso le llamaremos Análisis Discriminante Descriptivo (ADD). En la sección siguiente se presentarán las definiciones y resultados concernientes al ADD. En la sección 3 se halla un algoritmo a partir del cual se desarrolló la implementación computacional tal como se explica en la sección 4. Por último en la sección 5, se usa un ejemplo para ilustrar la aplicación del software y algunos aspectos metodológicos del ADD.

\section{Propiedades y resultados del ADD}

Se consideran $p$ variables continuas (variables explicativas) $x^{1}, \ldots, x^{p}$ observadas en una muestra $E$ de $n$ individuos. Cada individuo $i \in E$ se identifica con su vector de mediciones en $\mathbb{R}^{p}, x_{i}=\left(x_{i 1}, \ldots, x_{i p}\right)$ y cada variable $x^{j}$ con su vector de valores asumidos

$x^{j}=\left(x_{1 j}, x_{2 j}, \ldots, x_{n j}\right)^{t}$. La variable cualitativa $Y$ (a explicar) determina una partición de $E, P=\left\{E_{1}, \ldots, E_{r}\right\}$; cada clase $E_{i}$ se llama categoría o grupo a priori.

\subsection{Definiciones básicas}

Se supondrá que las variables $\left\{x^{1}, \ldots, x^{p}\right\} \subset \mathbb{R}^{n}$ son centradas.

$V=X^{t} D X$ es la matriz de correlaciones de las $p$ variables $x^{1}, \ldots, x^{p}$, donde $D=\operatorname{diag}\left(p_{i}\right)$ es la matriz diagonal de pesos de los individuos y $X=\left[x^{1} \cdots x^{p}\right]$.

$W=\sum_{l=1}^{r} \sum_{i \in E_{l}} p_{i}\left(x_{i}-g_{l}\right)^{t}\left(x_{i}-g_{l}\right)$ es la matriz de inercia intraclases, donde $g_{l}=\sum_{i \in E_{l}} \frac{p_{i}}{q_{l}} x_{i}$ con $q_{l}=\sum_{i \in E_{l}} p_{i}$ el peso de la clase $E_{l}$.

$B=\sum_{l=1}^{r} q_{l} g_{l}^{t} g_{l}$ es la matriz de inercia interclases. 
$\operatorname{inter}\left(x^{j}\right)=\sum_{l=1}^{r} q_{l} g_{j l}^{2}$ es la varianza interclases de la variable $x^{j}=\left(x_{1 j}, \ldots, x_{n j}\right)^{t}$ con respecto a la partición $P=\left\{E_{1}, \ldots, E_{r}\right\}$, donde $g_{j l}$ es la entrada $j$ de $g_{l}$. Por su parte la varianza intraclases de $x^{j}$ es: $\operatorname{intra}\left(x^{j}\right)=\sum_{l=1}^{r} \sum_{i \in E_{l}} p_{i}\left(x_{i}-g_{j l}\right)^{2}$.

Son bien conocidas las siguientes relaciones: ([2])

\section{Teorema 1:}

1. $B=C_{g}^{t} D_{q} C_{g}$, donde $C_{g}$ es la matriz $r \times p$ cuyas filas son $g_{1}, \ldots, g_{r}$ y $D_{q}=\operatorname{diag}\left(q_{l}\right)$.

2. $0=\sum_{l=1}^{r} q_{l} g_{l}$. Es decir, $C_{g}$ es de rango $\leq r-1$.

3. $\operatorname{rango}\left(C_{g}\right)=\operatorname{rango}(B) \leq r-1$ y si $r=2$, entonces $\operatorname{rango}(B)=1$.

4. $V=B+W$.

5. $\operatorname{var}(z)=\alpha^{t} V \alpha=\alpha^{t} W \alpha+\alpha^{t} B \alpha$ para todo $z=X \alpha \in \mathbb{R}^{n}$.

6. $\operatorname{intra}(z)=\alpha^{t} W \alpha$ e inter $(z)=\alpha^{t} B \alpha$.

\subsection{Cálculo de las funciones discriminantes: un ACP particular}

El objetivo principal del ADD consiste en determinar $m$ variables $z^{1}, \ldots, z^{m}$ llamadas funciones discriminantes, que poseen (y son determinadas por) las propiedades siguientes $(\operatorname{ver}[2])$ :

1. Cada $z^{j} \in \mathbb{R}^{p}$ es una combinación lineal de las $p$ variables. Esto es,

$$
z^{j}=\sum_{s=1}^{p} \alpha_{j s} x^{s}=X \alpha_{j},
$$

donde $\alpha_{j}=\left(\alpha_{j 1}, \ldots, \alpha_{j p}\right)^{t} ; j=1, \ldots, m$.

2. Las variables $z^{j}$ son $D$ ortonormadas. Es decir, no correlacionadas y de varianza 1.

3. Los valores de cada variable $z^{j}$ en los individuos de un mismo grupo, deben ser lo más próximos posible. Es decir, se debe minimizar intra $\left(z^{j}\right)$ (la varianza intraclases).

4. Los valores de cada variable $z^{j}$ en los individuos pertenecientes a clases distintas, deben ser lo más diferentes posible. Esto es, se debe maximizar $\operatorname{inter}\left(z^{j}\right)$ (la varianza interclases).

De acuerdo con la ecuación $1=\operatorname{Var}(z)=\alpha^{t} V \alpha=\alpha^{t} B \alpha+\alpha^{t} W \alpha$ se ve que las propiedades 3. y 4. de las funciones discriminantes son equivalentes:

$$
\max \left\{\alpha^{t} B \alpha \mid \alpha^{t} V \alpha=1\right\} \Leftrightarrow \min \left\{\alpha^{t} W \alpha \mid \alpha^{t} V \alpha=1\right\} .
$$


Diremos que $\left(\alpha_{1}, \lambda_{1}\right)$ es la primera solución del problema de máximo (salvo por el signo de $\left.\alpha_{1}\right)$ si $\lambda_{1}=\alpha_{1}^{t} B \alpha_{1}=\max \left\{\alpha^{t} B \alpha \mid \alpha^{t} V \alpha=1\right\}$. La primera variable discriminante es $z_{1}=X \alpha_{1}$. Las otras variables discriminantes se obtienen resolviendo el problema de máximo secuencialmente, con restricción de $V$ - ortogonalidad sobre $\alpha$. Esto es, $\left(\alpha_{k}, \lambda_{k}\right)$ es la $k$-ésima solución si

$$
\lambda_{k}=\alpha_{k}^{t} B \alpha_{k} \max \left\{\alpha^{t} B \alpha \mid \alpha^{t} V \alpha=1, \alpha^{t} V \alpha_{s}=0, s=1, \ldots, k-1\right\} .
$$

donde $\operatorname{los} \alpha_{1}, \ldots, \alpha_{k-1}$ fueron previamente calculados. Así, $\lambda_{k}$ es la inercia interclases de la $k$-ésima variable discriminante. Nótese que $\lambda_{k} \in[0,1]$.

Se va a probar que las variables $z^{j}$ se obtienen a partir de un ACP y que las representaciones bidimensionales de los individuos, de las clases y de las variables son consecuencia de este resultado. Estas representaciones ayudan a verificar si las variables discriminan las clases a priori y si es posible describirlas en términos de las variables originales.

Teorema 2: Sea $X$ de rango $p$-es decir, $V$ es invertible-. Si $\beta_{1}, \ldots, \beta_{t}$ son los vectores propios $V^{-1}$ - ortonormados del ACP del triplete $\left(C_{g}, V^{-1}, D_{q}\right)$, entonces las variables discriminantes son $z^{j}=X V^{-1} \beta_{j}, j=1, \ldots, t$.

Prueba: Sea $\beta=V \alpha$ entonces $\|\alpha\|_{V}=\|\beta\|_{V^{-1}}$ e inter $(z)=\alpha^{t} B \alpha=\beta^{t} V^{-1} B V^{-1} \beta$.

Es claro entonces que

$$
\max \left\{\alpha^{t} B \alpha \mid\|\alpha\|_{V}=1, \alpha \in \mathbb{R}^{p}\right\}=\max \left\{\beta^{t} V^{-1} B V^{-1} \beta \mid\|\beta\|_{V^{-1}}=1, \beta \in \mathbb{R}^{p}\right\} .
$$

Por otra parte, $\beta^{t} V^{-1} B V^{-1} \beta=\beta^{t} V^{-1}\left(C_{g}^{t} D_{q} C_{g}\right) V^{-1} \beta$ es la inercia de la nube de baricentros de las clases (es decir, las filas de la matriz $C_{g}$ ), $V^{-1}$ proyectada sobre la recta determinada por $\beta$.

De lo anterior es claro que los vectores propios $V^{-1}$ ortonormados del ACP del triplete $\left(C_{g}, V^{-1}, D_{q}\right)$ proveen la solución al problema de máximo. Sean $\beta_{1}, \ldots, \beta_{t}$ dichos vectores propios asociados a los valores propios $\lambda_{1} \geq \cdots \geq \lambda_{t}>0$.

Definiendo $\alpha_{j}=V^{-1} \beta_{j}$ resulta que las variables $z^{j}=X \alpha_{j}=X V^{-1} \beta_{j}, j=1, \ldots, t$ son $D$ - ortonormadas y satisfacen las otras propiedades que caracterizan las variables discriminantes ${ }^{1}$.

Cada valor propio $\lambda_{l}$ se llama poder discriminante y el vector propio correspondiente $\beta_{l}$, eje discriminante. Los ejes discriminantes son entonces los ejes de máxima inercia de la nube de baricentros. En este sentido decimos que son los ejes que más discriminan los grupos a priori.

El siguiente teorema tiene un interés práctico en la implementación computacional del ADD. Se sabe que el proceso de diagonalización de una matriz representa un esfuerzo computacional significativo. Para lograr más eficiencia en ese proceso se debe procurar diagonalizar siempre una matriz simétrica del menor tamaño posible, aún cuando posteriormente se deban hacer ciertas transformaciones. El teorema 3 nos garantiza que esto siempre es posible en ADD [4].

\footnotetext{
${ }^{1}$ Mediante un procedimiento similar al empleado en la prueba del teorema 2, se pueden deducir las funciones discriminantes resolviendo el problema de máximo $\max \left\{\frac{u^{t} B u}{u^{t} W u} \mid u \neq 0\right\}$, el cual es equivalente a: $\max \left\{v^{t} B v \mid v^{t} W v=1\right\}$.
} 
Teorema 3: Sea $C=C_{g}^{t} D_{q}^{\frac{1}{2}}, p \times r$.

1. Se tiene que $B=C C^{t}$

2. Sean $w_{1}, \ldots, w_{t}$ tales que $C^{t} V^{-1} C w_{j}=\lambda_{j} w_{j}$ con $\lambda_{j} \neq 0$ y $w_{j}^{t} I_{r} w_{s}=\delta_{j s}$. Entonces existen $\beta_{1}, \ldots, \beta_{t}$ tales que $B V^{-1} \beta_{j}=\lambda_{j} \beta_{j}$ y $\beta_{j}^{t} V^{-1} \beta_{s}=\delta_{j s}$.

3. Recíprocamente, sean $\beta_{1}, \ldots, \beta_{t}$ tales que $B V^{-1} \beta_{j}=\lambda_{j} \beta_{j}$ con $\lambda_{j} \neq 0$ y $\beta_{j}^{t} V^{-1} \beta_{s}=\delta_{j s}$. Entonces existen $w_{1}, \ldots, w_{t}$ tales que $C^{t} V^{-1} C w_{j}=\lambda_{j} w_{j}$ con $w_{j}^{t} I_{r} w_{s}=\delta_{j s}$.

4. Existe una matriz $S$ tal que $S B V^{-1} S^{-1}=H B H$ donde $H=U \Delta^{-\frac{1}{2}} U^{t}$ con $U=\left(u_{1}, \ldots, u_{p}\right), \Delta=\operatorname{diag}\left(\mu_{i}\right)$ y $u_{1}, \ldots, u_{p}$ son los vectores propios de $V$ asociados a $\mu_{1} \geq \cdots \geq \mu_{p}>0$. Los vectores propios de $B V^{-1}, V^{-1}$ ortonormados son: $\beta_{j}=H^{-1} w_{j}$ donde los $w_{j}$ son los vectores propios de $H B H, I_{p}$ ortonormados.

Prueba: 2. Definiendo $\beta_{j}=\frac{C w_{j}}{\sqrt{\lambda_{j}}}$ se obtiene el resultado. 3. Definiendo $w_{j}=\frac{1}{\sqrt{\lambda_{j}}} C^{t} V^{-1} \beta_{j}$ se obtiene el resultado. 4. Se sabe que $V^{-1}=\left(U \Delta^{-\frac{1}{2}} U^{t}\right)^{2}=H^{2}$ por lo tanto $B V^{-1} \sim$ $H B V^{-1} H^{-1}=H B H$. De aquí sigue que $B V^{-1} \beta_{j}=\lambda_{j} \beta_{j}$ con $\beta_{j}=H^{-1} w_{j}$. Además,

$$
\beta_{j} V^{-1} \beta_{s}=w_{j}^{t} H^{-1} V^{-1} H w_{s}=w_{j}^{t} I_{p} w_{s}=\delta_{j s} .
$$

\subsection{Representaciones en ADD}

A partir de los resultados obtenidos con el teorema 2, se pueden construir las siguientes representaciones bidimensionales para el caso de más de dos grupos a priori. Es decir, $r>2$.

\subsubsection{Representación de los grupos a priori}

Hemos visto que el ADD se puede interpretar como la búsqueda de los ejes (en $\mathbb{R}^{p}$ ) más discriminantes de los grupos a priori, en el sentido de maximización de la inercia interclases (teorema 2). Estos ejes son los vectores propios del ACP de $\left(C_{g}, V^{-1}, D_{q}\right)$, lo que nos permite al mismo tiempo calcular las funciones discriminantes. Para obtener las representaciones bidimensionales de los baricentros de los grupos, se proyectan éstos, $V^{-1}$ - ortogonalmente sobre los planos principales del ACP.

De lo anterior sigue que la coordenada del baricentro $g_{l}$ del grupo $E_{l}$, sobre el eje $j$ - ésimo es: $\operatorname{coord}_{j}\left(g_{l}\right)=g_{l} V^{-1} \beta_{j}$.

\subsubsection{Representación de las variables como son definidas por los grupos a priori}

Las columnas de la matriz $C_{g}$ representan las variables tal como son determinadas por los grupos a priori ya que cada columna $y^{j}$ de $C_{g}$ es el vector $\left(g_{j 1}, \ldots, g_{j r}\right)$, donde $g_{j l}$ es el promedio de la variable $x^{j}$ en el grupo $E_{l}$. Se les llamará variables promedio. 
Por las fórmulas de dualidad del ACP se sabe que las columnas de la matriz $\left(\beta_{1} \ldots \beta_{t}\right) D_{\sqrt{\lambda}}$ son las coordenadas de las columnas $y^{j}$ de $C_{g}$. Es decir, $\operatorname{coord}_{s}\left(y^{j}\right)=\sqrt{\lambda_{s}} \beta_{j s}$ para $j=1, \ldots, p$ y $s=1, \ldots, t$.

Superponiendo el gráfico de los grupos a priori y el de las variables promedio, es posible analizar la influencia de las variables en la determinación de los grupos a priori.

\subsubsection{Representación de los individuos}

Los individuos se proyectan en suplementario sobre los ejes discriminantes. Es decir, sobre los ejes principales del ACP de $\left(C_{g}, V^{-1}, D_{q}\right)$. Sea $x_{i}$ el $i$-ésimo individuo, su coordenada sobre el $j$ - ésimo eje es $\operatorname{coord}_{j}\left(x_{i}\right)=x_{i} V^{-1} \beta_{j}$.

Es claro que el vector de coordenadas de los individuos sobre el $j$ - ésimo eje es la función discriminante $z^{j}$.

\subsubsection{Representación de las variables}

Las variables se representan en el sistema $D$ - ortonormado determinado por las variables discriminantes. La coordenada de la variable $x^{j}$ (columna $j$ - ésima de $X$ ) sobre el eje $s-$ ésimo es: $\operatorname{coord}_{s}\left(x^{j}\right)=x^{j} D z^{s}=\beta_{j s}$. En efecto, como $z^{s}=X V^{-1} \beta_{s}$ entonces el vector de coordenadas de las variables en la dirección de la variable discriminante $z^{s}$ es,

$$
X^{t} D z^{s}=X^{t} D X V^{-1} \beta_{s}=\beta_{s} .
$$

Si las variables son estandarizadas (varianza igual a 1) entonces $\operatorname{coord}_{s}\left(x^{j}\right)=\operatorname{corr}\left(x^{j}, z^{s}\right)$ $\mathrm{y}$ las variables se pueden representar como en ACP normado, en un círculo de correlaciones.

\subsubsection{El caso de dos grupos a priori}

Cuando solo hay dos grupos a priori, las representaciones se simplifican ya que el rango de $B$ (y por tanto el de $B V^{-1}$ ) vale 1 . En este caso $g_{2}-g_{1}$ es un vector propio de $B V^{-1}$ asociado al único valor propio $q_{1} q_{2}\left\|g_{2}-g_{1}\right\|_{V^{-1}}$. Se puede leer una prueba de este resultado por ejemplo en [7]. En consecuencia, las representaciones tanto de los individuos y baricentros como de las variables, se hacen sobre una recta.

\subsubsection{Indices de calidad}

La calidad de la discriminación en un subespacio principal de dimensión $q$ es el porcentaje de inercia explicada:

$$
100 \frac{\sum_{j=1}^{q} \lambda_{j}}{\text { Inercia Total }}=100 \frac{\sum_{j=1}^{q} \lambda_{j}}{\sum_{j=1}^{t} \lambda_{j}}
$$

donde $t$ es el número de valores propios positivos del ACP de $\left(C_{g}, V^{-1}, D_{q}\right)$.

La calidad de la representación de los baricentros y de los individuos en cada eje discriminante se mide por medio de los cosenos cuadrados de la misma forma como se hace en ACP. Las fórmulas para el cálculo de estos cosenos son: 
- Baricentros:

$$
\cos ^{2}\left(g_{l}, s\right)=\frac{\left\|\operatorname{Pr}_{s}\left(g_{l}\right)\right\|_{V^{-1}}^{2}}{\left\|g_{l}\right\|_{V^{-1}}^{2}}=\frac{\left\|\left(g_{l} V^{-1} \beta_{s}\right) \beta_{s}\right\|_{V^{-1}}^{2}}{g_{l} V^{-1} g_{l}^{t}}=\frac{\left(g_{l} V^{-1} \beta_{s}\right)^{2}}{g_{l} V^{-1} g_{l}^{t}} .
$$

- Individuos:

$$
\cos ^{2}(i, s)=\frac{\left\|\operatorname{Pr}_{s}\left(x_{i}\right)\right\|_{V^{-1}}^{2}}{\left\|x_{i}\right\|_{V^{-1}}^{2}}=\frac{\left\|\left(x_{i} V^{-1} \beta_{s}\right) \beta_{s}\right\|_{V^{-1}}^{2}}{x_{i} V^{-1} x_{i}^{t}}=\frac{\left(x_{i} V^{-1} \beta_{s}\right)^{2}}{x_{i} V^{-1} x_{i}^{t}} .
$$

\section{Algoritmo para la implementación computacional del ADD}

En esta implementación se usaron las mismas notaciones introducidas en el texto precedente y se asume que todos los individuos tienen peso igual: $p_{i}=\frac{1}{n}, \quad i=1,2, \ldots, n$. El número de grupos a priori debe ser mayor que 2, es decir $r>2$.

Para el cálculo de los cosenos de individuos y baricentros, se utilizaron las identidades:

$$
x_{i} V^{-1} x_{i}^{t}=\sum_{h=1}^{t}\left[\operatorname{coord}_{h}\left(x_{i}\right)\right]^{2} \quad \text { y } g_{l} V^{-1} g_{l}^{t}=\sum_{h=1}^{t}\left[\operatorname{coord}_{h}\left(g_{l}\right)\right]^{2} .
$$

1. Operación de centraje y reducción:

1.1 Para $j=1, \ldots, p$ se calcula $\bar{x}^{j}=\frac{1}{n} \sum_{i=1}^{n} x_{i j}$ y $\sigma_{j}^{2}=\frac{1}{n} \sum_{i=1}^{n} x_{i j}^{2}-\left(\bar{x}^{j}\right)^{2}$.

1.2 Para $i=1, \ldots, n$ y $j=1, \ldots, p$ se sustituye la entrada $x_{i j}$ de $X$ por $x_{i j}-\bar{x}^{j}$ para el centraje, y por $\frac{x_{i j}-\bar{x}^{j}}{\sigma_{j}}$ para la reducción.

2. Baricentros de los grupos: para $l=1, \ldots, r ; j=1, \ldots, p$ calcular $g_{j l}=\frac{1}{n_{l}} \sum_{i \in E_{l}} x_{i j}$ donde $n_{l}=\left|E_{l}\right|$.

3. Calcular $V=\frac{1}{n} X^{t} X=\left(v_{i j}\right)_{p \times p}$, donde $v_{i j}=\frac{1}{n} \sum_{s=1}^{n} x_{s i} x_{s j}$ para $i, j=1, \ldots, p$.

4. Calcular $V^{-1}$ :

4.1 Para $V$ calcular sus valores propios $\mu_{1} \geq \cdots \geq \mu_{p}>0$ y los vectores propios correspondientes $u_{1}, \ldots, u_{p}, I_{p}$ - ortonormados.

4.2 Sea $V^{-1}=\left(t_{i j}\right)$; para $i, j=1, \ldots, p$; calcular $t_{i j}=\sum_{s=1}^{p} \frac{1}{\mu_{s}} u_{i s} u_{j s}$, con $u_{s}^{t}=\left(u_{1 s}, \ldots, u_{p s}\right)$.

5. Sea $C$ la matriz definida en el teorema 3. Si $r \leq p$ calcular $A=C^{t} V^{-1} C$, sus valores propios no nulos, los vectores propios $I_{r}$ - ortonormados, y los $\beta_{j}$ correspondientes (ver la prueba del teorema 3, parte 2.):

5.1 Para $l, k=1, \ldots, r$ calcular

$$
\left(C^{t} V^{-1} C\right)_{l k}=\frac{\sqrt{n_{l} n_{k}}}{n} \sum_{b=1}^{p} g_{b k}\left[\sum_{f=1}^{p} g_{f l} t_{f b}\right]
$$


5.2 Calcular los valores propios $\lambda_{1} \geq \cdots \geq \lambda_{t}>0$ de $A$ y los vectores propios correspondientes $w_{1}, \ldots, w_{t}, I_{r}$ ortonormados.

5.3 Calcular $\beta_{j}=\frac{C w_{j}}{\sqrt{\lambda_{j}}}$ : Es decir, para $j=1, \ldots, t$ y $s=1, \ldots, p$ calcular:

$$
\beta_{s j}=\frac{1}{\sqrt{n \lambda_{j}}} \sum_{k=1}^{r} \sqrt{n_{k}} g_{s k} w_{k j} .
$$

6. Si $r>p$ calcular $A=H B H$ y sus valores propios no nulos, sus vectores propios $I_{p}$ - ortonormados y los $\beta_{h}$ correspondientes (ver teorema 3 , parte 4 .):

6.1 Cálculo de $B$ y $H$ : para $i, j=1, \ldots, p$ calcular

6.1.1 $B_{i j}=\frac{1}{n} \sum_{s=1}^{r} n_{s} g_{i s} g_{j s}$

6.1.2 $H_{i j}=\sum_{k=1}^{p} \frac{1}{\sqrt{\mu_{k}}} u_{i k} u_{k j}$

6.2 Cálculo de $H B$ y $H B H$ :

6.2.1 para $i, j=1, \ldots, p$ calcular $(H B)_{i j}=\sum_{s=1}^{p} H_{i s} B_{s j}$

6.2 .2 para $i, j=1, \ldots, p$ calcular $(H B H)_{i j}=\sum_{s=1}^{p}(H B)_{i s} H_{s j}$

6.3 Calcular los valores propios $\lambda_{1} \geq \cdots \geq \lambda_{t}>0$ de $A$ y los vectores propios correspondientes $w_{1}, \ldots, w_{t}, I_{p}$ - ortonormados.

6.4 Cálculo de $\beta_{j}=H^{-1} w_{j}:$ para $j=1, \ldots, t, s=1, \ldots, p$ calcular $\beta_{s j}=\sum_{k=1}^{p} w_{k j}\left[\sum_{h=1}^{p} \sqrt{\mu_{h}} u_{s h} u_{h k}\right]$.

7. Cálculo de coordenadas:

7.1 Baricentros: para $s=1, \ldots, t ; l=1, \ldots, r$;

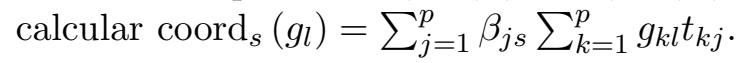

7.2 Individuos: para $s=1, \ldots, t ; i=1, \ldots, n$; calcular $\operatorname{coord}_{s}\left(x_{i}\right)=\sum_{j=1}^{p} \beta_{j s} \sum_{k=1}^{p} x_{i k} t_{k j}$.

7.3 Variables: para $s=1, \ldots, t ; j=1, \ldots, p$; calcular $\operatorname{coord}_{z^{s}}\left(x^{j}\right)=\beta_{j s}$.

8. Cálculo de los cosenos cuadrados:

8.1 Individuos: para $s=1, \ldots, t ; i=1, \ldots, n$; calcular $\cos ^{2}(i, s)=\frac{\left[\operatorname{coord}_{s}\left(x_{i}\right)\right]^{2}}{\sum_{h=1}^{t}\left[\operatorname{coord}_{h}\left(x_{i}\right)\right]^{2}}$.

8.2 Baricentros: para $s=1, \ldots, t ; l=1, \ldots, r$; calcular $\cos ^{2}(l, s)=\frac{\left[\operatorname{coord}_{s}\left(g_{l}\right)\right]^{2}}{\sum_{h=1}^{t}\left[\operatorname{coord}_{h}\left(g_{l}\right)\right]^{2}}$. 


\section{Acerca de la implementación [5]}

El algoritmo anterior fue implementado en lenguaje $\mathrm{C}++$ utilizando la Programación Orientada a Objetos. Se programaron las clases Matriz, TablaDatos, MatrizV, MatrizA, Plano-Principal, CirculoCorrelacion, CosenosIndividuos y CosenosVariables. La clase Matriz se encarga de aspectos comunes a todas las clases, como son manipular la matriz en memoria, guardarla y recuperarla de disco, además tiene métodos para calcular estadísticas básicas como la media, la correlacion, la desviación estándar entre otras. La clase TablaDatos hereda de la clase Matriz y se encarga de manipular la tabla datos y la variable cualitativa. Tiene métodos (además de los heredados) para centrar y reducir la tabla, para calcular y almacenar los baricentros de los grupos (paso 2 del algoritmo). La clase MatrizV hereda de la clase Matriz y se encarga de los pasos 3 y 4 del algoritmo, es decir, tiene métodos para calcular $V, V^{-1}$ y los valores y vectores propios de $V$. La clase MatrizA hereda de clase MatrizV y se encarga de los pasos 5 y 6 del algoritmo por lo que tiene métodos para calcular tanto la matriz $A$ como los $\beta_{j}$. Los métodos para calcular los valores y vectores propios los hereda de la clase MatrizA. La clase PlanoPrincipal hereda de la clase Matriz y tiene métodos para calcular las coordenadas de los individuos y de los baricentros (pasos 7.1 y 7.2 del algoritmo), así como para generar el plano principal en formato $\mathrm{AT}_{\mathrm{E} X} \mathrm{y}$ en formato Windows. Similarmente, la clase CirculoCorrelacion hereda de la clase Matriz y tiene métodos para calcular las coordenadas de las variables (paso 7.3 del algoritmo) y para generar el círculo de correlación en formato $\mathrm{IAT}_{\mathrm{EX}} \mathrm{y}$ en formato Windows. Finalmente, las clases CosenosIndividuos y CosenosVariables se encargan de los pasos 8.1 y 8.2 del algoritmo. Es decir, calculan los cosenos cuadrados de los individuos y los baricentros.

\section{$5 \quad$ Ejemplo ilustrativo}

Esta sección tiene el propósito de ilustrar el uso del software y de mostrar los productos que se pueden obtener mediante su aplicación.

\subsection{Sobre los datos de contaminación de ríos}

Vamos a considerar 13 variables relacionadas con la contaminación de aguas (entre paréntesis se coloca el nombre abreviado de la variable ): Fosfato (FOS), Nitratos (NITA), Calidad del agua (CAL), Sólidos totales (STOT), Acidez (PH), Manganeso (MN), Zinc (ZN), Sólidos Sedimentales (SS), Alcalinidad (ALCA), Cloro (CL), Caudal (CAU), Demanda Bioquímica de Oxígeno (DBO) y Porcentaje de Saturación de Oxígeno (PORS). Estas variables fueron medidas en los ríos que forman el embalse La Garita, en 9 puntos de muestreo que corresponden a la Presa, los ríos Alajuela, Ciruelas, Virilla y Quebrada Soto; tres en el Embalse (Orilla, Centro y Salida) y uno en el Desfogue-Garita. Se hace una medición por estación: Verano (V), Verano-Invierno (VI), Invierno (I) e InviernoVerano (IV). El nombre de un punto de muestreo cualquiera se forma con las primeras letras del nombre del sitio seguido por el nombre abreviado de la estación. Por ejemplo, 
PV es la Presa en Verano, VVI es el río Virilla en Verano Invierno y EOV es el Embalse Orilla en Verano.

La tabla de datos contiene el promedio de cada variable para cada caso "sitio-estación" dando como resultante una matriz de datos de 36 filas (sitio-estación) y 13 columnas (ver $[3])$.

Los grupos a priori que se quieren discriminar son los definidos por las 4 estaciones: V, VI, I e IV. La composición de estos grupos se presenta en la tabla siguiente:

\begin{tabular}{|c|c|}
\hline G1 & PV - DV - EOV - ESV - ECV - AV - CV - QV - VV \\
\hline G2 & PVI - DVI - EOVI - ESVI - ECVI - AVI - CVI - QVI - VVI \\
\hline G3 & PI - DI - EOI - ESI - ECI - AI - CI - QI - VI \\
\hline G4 & PIV - DIV - EOIV - ESIV - ECIV - AIV - CIV - QIV - VIV \\
\hline
\end{tabular}

\subsection{Los archivos de datos y etiquetas}

Para hacer los cálculos el sistema necesita cuatro archivos tipo texto, los cuales se describen a continuación:

- El archivo de datos: debe tener extensión TXT. El primer registro (fila) debe contener dos números separados por blancos que indican respectivamente el número de individuos y variables. Los demás registros contienen los datos propiamente dichos. En el caso de los datos de contaminación de ríos el archivo tiene 37 registros y 13 columnas. Los primeros 4 registros del archivo son los siguientes:

3613

$\begin{array}{lllllllllllll}1.44 & 0.78 & 58.25 & 126.75 & 7.29 & 0.21 & 0.02 & 0.66 & 120.50 & 3.92 & 17.85 & 25.00 & 53.25\end{array}$

$2.701 .1362 .50195 .00 \quad 7.50 \quad 0.20 \quad 0.02 \quad 1.15 \quad 104.504 .10 \quad 28.20 \quad 90.70 \quad 77.00$

$\begin{array}{lllllllllllll}3.89 & 0.35 & 79.25 & 176.50 & 7.41 & 0.37 & 0.28 & 0.62 & 80.25 & 3.35 & 53.27 & 3.77 & 89.75\end{array}$

- El archivo de etiquetas de los individuos: debe tener extensión ETI (de ETiqueta de los Individuos). Consta de una sola columna que contiene en la primera posición un número que indica cuantos individuos hay. En las restantes posiciones vienen las etiquetas de los individuos. En el caso de los datos de contaminación de ríos el archivo tiene 37 registros. Los primeros 4 registros del archivo son los siguientes:

36

$\mathrm{PV}$

PVI

PI

- El archivo de etiquetas de las variables: debe tener extensión ETV (de ETiqueta de las Variables). Consta de una sola columna que contiene en la primera posición un número que indica cuantas variables hay. En las restantes posiciones vienen las etiquetas de las variables (las cuales no pueden tener espacios en blanco). En el caso de los datos de contaminación de ríos el archivo tiene 14 registros. Los primeros 4 registros del archivo son los siguientes: 
NITA

FOS

CAL

- El archivo de la variable cualitativa: debe tener extensión CUA (de variable CUAlitativa) y consta de una sola columna. En la primera posición contiene un número que indica cuantas clases hay. En las restantes posiciones se escribe un número entero que indica el número de clase a la que pertenece el individuo en cuestión. En el caso de los datos de contaminación de ríos el archivo tiene 37 registros. Los primeros 5 registros del archivo son los siguientes:

4
1
2
3
4

\subsection{El submenú Discriminante-Paso-a-Paso}

El módulo de Análisis Discriminante se integró al Programa Interactivo para Métodos de Análisis de Datos en su versión 3.0 (PIMAD 3.0). Se dispone de tres variantes para ejecutar los cálculos:

- El uso de los botones para conseguir los gráficos en pantalla, los cosenos de los individuos y de los baricentros.

- El submenú Discriminante-Directo con el cual se despliegan los gráficos en pantalla.

- El submenú Discriminante-Paso-a-Paso permite ejecutar el algoritmo de la sección 3 paso a paso a través de 16 opciones.

A continuación se describe cada opción del submenú Discriminante-Paso-a-Paso. Todos los archivos de 'salida' son tipo ASCII con extensión TXT.

1. Opción: Centraje y Reducción de la Tabla de Datos. Con esta opción se crea un archivo de nombre tabla.txt que contiene en columna las variables explicativas centradas y reducidas (es decir, estandarizadas).

2. Opción: Calcula los Baricentros. Calcula los baricentros de cada grupo a priori.

3. Opción: Calcular la matriz V. Crea un archivo de nombre mat_V.txt que contiene la matriz de correlaciones de las variables explicativas $(V)$.

4. Opción: Calcular los Vectores y Valores Propios de V. Crea dos archivos de nombres vectorp.txt y valorp.txt que contienen respectivamente los vectores propios $I_{13}$ ortonormados de $V$ y los valores propios correspondientes. 
5. Opción: Calcular la Matriz $\mathrm{V}^{\wedge}(-1)$. Crea un archivo de nombre vinv.txt que contiene la matriz inversa de $V$.

6. Opción: Calcular la Matriz A. Crea un archivo de nombre mat_A.txt que contiene la matriz $A$ (ver pasos 5. y 6 . del algoritmo). En nuestro ejemplo, este archivo es:

$$
A=\left(\begin{array}{rrrr}
0.547 & -0.223 & -0.260 & -0.064 \\
-0.223 & 0.654 & -0.223 & -0.207 \\
-0.260 & -0.224 & 0.650 & -0.166 \\
-0.064 & -0.207 & -0.166 & 0.436
\end{array}\right)
$$

7. Opción: Calcular los Vectores y Valores Propios de A. Crea dos archivos de nombres $\mathbf{v e c} \_\mathbf{p} \_$A.txt y val_p_A.txt que contienen respectivamente los vectores propios de $A, I_{5}$ ortonormados y los valores propios correspondientes (que son al mismo tiempo los valores propios positivos de $B V^{-1}$ ). Para nuestro ejemplo, estos valores propios son:

\begin{tabular}{|c|c|c|}
\hline$\lambda_{1}$ & $\lambda_{2}$ & $\lambda_{3}$ \\
\hline 0.9283 & 0.8617 & 0.4203 \\
\hline
\end{tabular}

El archivo de los correspondientes vectores propios de $A$, tiene la apariencia siguiente:

\begin{tabular}{|c|c|c|}
\hline$w_{1}$ & $w_{2}$ & $w_{3}$ \\
\hline 0.425 & 0.499 & -0.566 \\
\hline 0.309 & -0.808 & -0.038 \\
\hline-0.843 & -0.004 & -0.196 \\
\hline 0.109 & 0.313 & 0.800 \\
\hline
\end{tabular}

8. Opción: Calcular la matriz Beta. Con esta opción se calcula la matriz de vectores propios de $B V^{-1}, V^{-1}$ ortonormados y la guarda en un archivo de nombre beta.txt. Estos vectores propios son:

\begin{tabular}{|c|c|c|c|c|c|c|c|c|c|c|c|c|c|}
\hline$\beta_{1}$ & -0.39 & 0.60 & -0.89 & 0.14 & 0.25 & -0.29 & -0.18 & 0.24 & 0.32 & 0.19 & -0.33 & 0.45 & -0.50 \\
\hline$\beta_{2}$ & -0.19 & -0.73 & 0.10 & -0.53 & 0.06 & -0.31 & 0.13 & -0.34 & 0.28 & -0.26 & 0.04 & -0.69 & -0.17 \\
\hline$\beta_{3}$ & -0.27 & -0.18 & 0.25 & -0.21 & -0.05 & -0.30 & 0.81 & -0.00 & -0.41 & -0.17 & 0.41 & -0.14 & -0.26 \\
\hline
\end{tabular}

Aquí se presentan los $\beta_{j}$ en las filas, sin embargo debe notarse que en el archivo beta.txt los vectores $\beta_{1}, \ldots, \beta_{4}$ se almacenan en las columnas.

9. Opción: Calcular Componentes Principales y las Coordenadas de Baricentros.

Crea un archivo de nombre compone.txt que contiene las coordenadas de los individuos en los ejes determinados por los vectores propios de $B V^{-1}$. Los primeros 5 
registros de este archivo son:

\begin{tabular}{|l|c|c|c|}
\cline { 2 - 4 } \multicolumn{1}{c|}{} & Eje 1 & Eje 2 & Eje 3 \\
\hline PV & 1.326 & 0.589 & -0.751 \\
\hline PVI & 0.524 & -1.689 & -0.757 \\
\hline PI & -1.695 & -0.229 & 0.806 \\
\hline PIV & 0.340 & 0.737 & 3.656 \\
\hline AV & 0.745 & 1.444 & -2.288 \\
\hline
\end{tabular}

10. Opción: Graficar el Plano Principal. Despliega una ventana donde el usuario puede escoger dos ejes para representar simultáneamente los individuos y los baricentros de los grupos a priori. Opcionalmente el usuario puede escoger los individuos que desea representar y el tamaño del gráfico.

11. Opción: Generar Archivo $\mathbb{H T}_{\mathrm{E}} \mathrm{X}$ del Plano Principal. Crea un archivo tipo $\mathrm{AT}_{\mathrm{E}} \mathrm{X}$ con las coordenadas de los individuos en el plano principal escogido (plano discriminante). El número al final de las etiquetas indica el grupo a priori al que pertenece el individuo. Por ejemplo, QIV-4 en el extremo inferior del eje vertical indica que el individuo QIV pertenece a la clase a priori número 4. Para nuestro ejemplo el plano principal es:

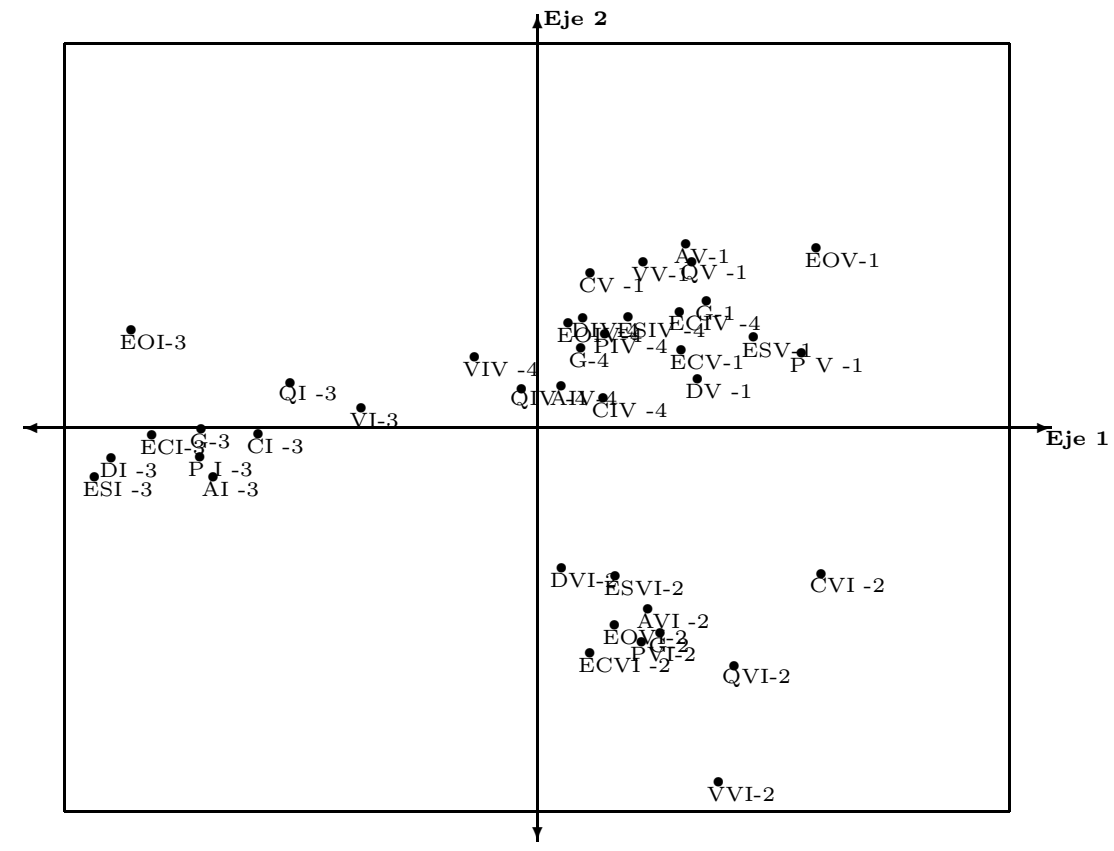


12. Opción: Calcular Principales Correlaciones. Crea un archivo de nombre princorr.txt que contiene las coordenadas de las variables explicativas centradas y reducidas, en los ejes determinados por las variables discriminantes.

13. Opción: Graficar el Círculo de Correlación. Despliega una ventana donde el usuario puede escoger dos ejes, las variables que desea representar y el tamaño del gráfico. Las coordenadas son tomadas del archivo princorr.txt.

14. Opción: Generar Archivo $\mathbb{H T}_{\mathrm{E}} \mathrm{X}$ del Círculo de Correlación. Crea un archivo tipo $\mathrm{AT}_{\mathrm{E}} \mathrm{X}$ con las coordenadas de las variables, para nuestro ejemplo el círculo de correlación es:

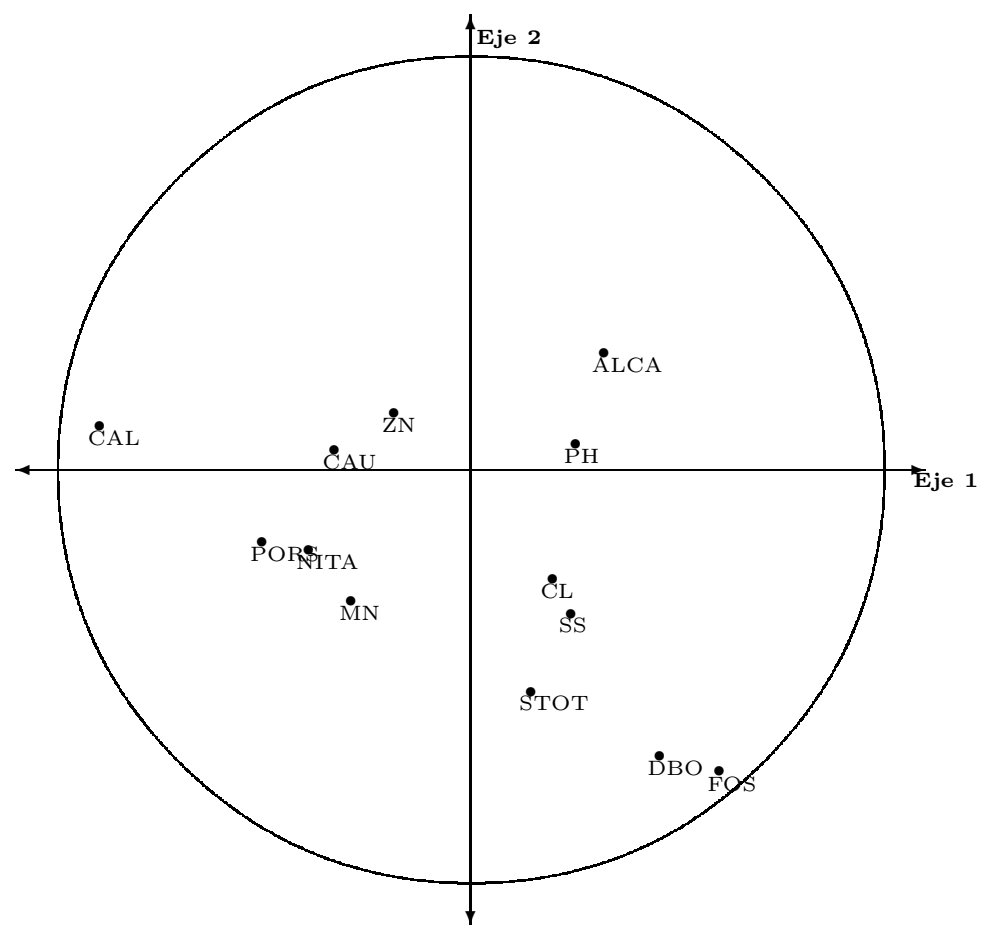

15. Opción: Calcula Cosenos Cuadrados de los Individuos. Crea un archivo de nombre cos_ind.txt que contiene los cuadrados de los cosenos del ángulo que forma cada individuo con su proyección $V^{-1}$ - ortogonal, sobre los ejes discriminantes. Los cinco primeros registros de cos_ind.txt son:

\begin{tabular}{|l|c|c|c|}
\cline { 2 - 4 } \multicolumn{1}{c|}{} & $\cos ^{2}(i, 1)$ & $\cos ^{2}(i, 2)$ & $\cos ^{2}(i, 3)$ \\
\hline PV & 0.659 & 0.130 & 0.211 \\
\hline PVI & 0.074 & 0.771 & 0.155 \\
\hline PI & 0.804 & 0.015 & 0.182 \\
\hline PIV & 0.008 & 0.039 & 0.953 \\
\hline AV & 0.070 & 0.265 & 0.665 \\
\hline
\end{tabular}


16. Opción: Calcula Cosenos Cuadrados de los Baricentros. Crea un archivo de nombre cos_bar.txt que contiene los cuadrados de los cosenos del ángulo que forma cada baricentro con su proyección $V^{-1}$ ortogonal, sobre los ejes discriminantes. Para nuestro ejemplo estos cosenos valen:

\begin{tabular}{|c|c|c|c|}
\cline { 2 - 4 } \multicolumn{1}{c|}{} & $\cos ^{2}\left(g_{l}, 1\right)$ & $\cos ^{2}\left(g_{l}, 2\right)$ & $\cos ^{2}\left(g_{l}, 3\right)$ \\
\hline G-1 & 0.241 & 0.332 & 0.427 \\
\hline G-2 & 0.128 & 0.870 & 0.002 \\
\hline G-3 & 0.949 & 0.000 & 0.051 \\
\hline G-4 & 0.016 & 0.131 & 0.853 \\
\hline
\end{tabular}

\section{References}

[1] Celeux, G.; Nakache, J.P. (1994) Analyse Discriminante sur Variables Qualitatives. Polytechnica, Paris.

[2] Diday, E.; Lemaire, J.; Pouget, J.; Testu, F. (1982) Eléments d'Analyse de Données. Dunod, Paris.

[3] González, J.; Morales, V. (1993) "Análisis multivariado de la calidad del agua: proyecto hidroeléctrico Ventanas-Garita", VI Congreso Internacional de Biomatemáticas, J. Oviedo et al. (Eds.), Universidad de Costa Rica: 227-236.

[4] Lebart, L.; Morineau, A.; Piron, M. (1994) Statistique Exploratoire Multidimensionnelle. Dunod, Paris.

[5] Rodríguez, O. (1997) Introducción a la Programación C++ para Ambiente Windows. Editorial Tecnológica de Costa Rica, Cartago, Costa Rica.

[6] Saporta, G. (1994) "Los métodos y las aplicaciones del credit-scoring", VII y VIII Simposios, W. Castillo \& J. Trejos (Eds.), Editorial de la Universidad de Costa Rica, San José: 103-109.

[7] Saporta, G. (1994) "Análisis discriminante", Métodos Matemáticos Aplicados a las Ciencias, VII y VIII Simposios, W. Castillo \& J. Trejos (Eds.), Editorial de la Universidad de Costa Rica, San José: 75-102.

[8] Saporta, G. (1980) L'Analyse des Données. Que sais-je?, Presses Universitaires de France, Paris. 